\title{
Luteinizing Hormone to Follicle-Stimulating Hormone Ratio Significantly Correlates With Androgen Level and Manifestations Are More Frequent With Hyperandrogenemia in Women With Polycystic Ovary Syndrome
}

\author{
Md. Shahed Morshed ${ }^{\mathrm{a}}$, Hurjahan Banu ${ }^{\mathrm{a}, \mathrm{b}}$, Nazma Akhtar ${ }^{\mathrm{a}}$, Tania Sultana ${ }^{\mathrm{a}}$, Afroza Begumª, \\ Moriom Zamilla a, Sadiqa Tuqan a, Sukanti Shah ${ }^{a}$, Ahmed Hossaina, Shazia Afrinea, \\ Emran Ur Rashid ${ }^{\text {a }}$, Iffat Ara Jahan ${ }^{\text {a }}$, M.A. Hasanat ${ }^{\mathrm{a}}$
}

\begin{abstract}
Background: Altered luteinizing hormone to follicle-stimulating hormone (LH-FSH) ratio and hyperandrogenism are two important pathophysiological characteristics of polycystic ovary syndrome (PCOS). The aim of this study was to evaluate the relationship of LHFSH ratio with androgen in women with PCOS.

Methods: This cross-sectional study included 550 newly detected reproductive aged women with PCOS (mean \pm standard deviation (SD): age $23.14 \pm 4.80$ years; body mass index (BMI) $27.64 \pm 5.34$ $\mathrm{kg} / \mathrm{m}^{2}$ ) according to revised Rotterdam criteria. Relevant clinical history, physical examination and ultrasonogram of ovaries were done for each participant. Fasting serum LH, FSH and total testosterone (TT) were measured by chemiluminescent microparticle immunoassay from blood collected during follicular phase of menstrual cycle.
\end{abstract}

Results: More than two-thirds ( $\mathrm{n}=389$, about $71 \%$ ) had altered LHFSH ratio (cut-off $>1.0)$ and about $43 \%(n=234)$ had hyperandrogenemia (TT $>46 \mathrm{ng} / \mathrm{dL}$ ). Frequency of none of the clinical variables or ovarian morphology differed statistically between the groups with or without altered LH-FSH ratio (NS for all). Serum TT and LH-FSH ratio were positively correlated $(\mathrm{r}=0.139, \mathrm{P}=0.001)$. However, menstrual irregularity $(\mathrm{P}=0.002)$, polycystic ovaries $(\mathrm{P}=0.045)$, diabetes mellitus $(\mathrm{P}=0.017)$, obesity $(\mathrm{P}=0.009)$ and hirsutism $(\mathrm{P}<0.001)$ were higher in frequency in the hyperandrogenic group. Serum TT had predictive association with altered LH-FSH ratio (odds ratio (OR) $(95 \%$ confidence interval $(\mathrm{CI})): 1.09(1.02-1.16), \mathrm{P}=0.02)$ and LH-FSH had predictive association with hyperandrogenemia (OR (95\% CI): 1.15 (1.03 - 1.28), $\mathrm{P}=0.02)$ in women with PCOS.

Manuscript submitted November 29, 2020, accepted December 15, 2020 Published online February 3, 2021

aPCOS Study Group, Department of Endocrinology, Bangabandhu Sheikh Mujib Medical University (BSMMU), Dhaka 1000, Bangladesh

${ }^{b}$ Corresponding Author: Hurjahan Banu, Department of Endocrinology, Bangabandhu Sheikh Mujib Medical University (BSMMU), Shahbag, Dhaka 1000, Bangladesh. Email: dr.hurjahan_banu@yahoo.com

doi: https://doi.org/10.14740/jem716
Conclusions: Serum LH-FSH ratio and androgenemia significantly correlate in women with PCOS. However, manifestations are more frequent with hyperandrogenemia rather than altered LH-FSH ratio.

Keywords: PCOS; LH-FSH ratio; Androgen; Hyperandrogenemia

\section{Introduction}

Polycystic ovary syndrome (PCOS) is one of the growing endocrine healthcare burdens of reproductive women throughout the world. The usual worldwide prevalence is $8-13 \%$ with highest prevalence reported from Indian Asian women (52\%) $[1,2]$. As a heterogeneous condition with several phenotypes, a criterion for diagnosing PCOS is difficult to be set. Currently it is diagnosed mostly on the basis of revised Rotterdam criteria (two out of three: ovulatory dysfunction, hyperandrogenism/hyperandrogenemia and polycystic ovarian morphology (PCOM) on ultrasonography) that were also recommended recently by an international evidence-based guideline of PCOS $[1,3]$. The pathophysiology of PCOS is still unresolved. Recent studies suggest that the brain is an important regulator as well as an affected organ in PCOS (the brain phenotype). The brain containing several receptors (androgen, estrogen and progesterone) and neurons (gonadotropin-releasing hormone, gamma amino-butyric acid (GABA) and kisspeptinneurokinin-dynorphin) with their neurotransmitters (serotonin, GABA and glutamate) produces an increased pulse frequency of gonadotropin. As a result, an elevated luteinizing hormone (LH) secretion compared to follicle-stimulating hormone (FSH) is observed among patients with PCOS [4]. This altered LH-FSH ratio is responsible for increased ovarian androgen production. On the other hand, hyperandrogenemia reduces the negative feedback of estrogen to hypothalamus leading to increased pulse frequency of LH. So, a vicious cycle is produced that is responsible for several clinical manifestations of PCOS including hyperandrogenism [5]. This study was done to see the relationship of LH-FSH ratio with clinical and biochemical androgen status in Bangladeshi women with PCOS. 


\section{Materials and Methods}

This was a cross-sectional observational study. A total of 550 patients were recruited consecutively by purposive sampling from the Department of Endocrinology, BSMMU during the period of January 2018 to August 2019. The study protocol was approved by the institutional review board of BSMMU (No. BSMMU/2017/292). This study was conducted in compliance with the ethical standards of the responsible institution on human subjects as well as with the Helsinki Declaration.

Females of reproductive age were included by revised Rotterdam criteria after excluding several similar endocrine disorders (hypothyroidism, hyperprolactinemia, non-classical congenital adrenal hyperplasia, primary amenorrhea, etc.) by clinical examination and biochemical investigations as required. Data were collected in a pretested semi-structured data collection sheet. Personal and family histories related to PCOS were taken. Physical examination included height, weight, waist circumference (WC), blood pressure and hirsutism measured by modified Ferriman-Gallwey (mF-G) score. Body mass index (BMI) was considered as measure of obesity and obesity was defined by BMI $\geq 25 \mathrm{~kg} / \mathrm{m}^{2}$ [6]. The cut-off values to define central obesity, hypertension and significant hirsutism were $80 \mathrm{~cm}, 140 / 90 \mathrm{~mm} \mathrm{Hg}$ and $\mathrm{mF}-\mathrm{G}$ score 8, respectively [68]. Ultrasonography (Toshiba Aplio 500 imaging machine) for ovarian volume and specific sized follicle number was done during follicular phase by trans-abdominal or trans-vaginal approach as per marital status of the participants.

\section{Sampling and analytic methods}

Women were asked to come during the menstrual period of days 2 - 5 after a spontaneous menstruation or on a convenient day in case of amenorrhea. About $10 \mathrm{~mL}$ venous blood was collected during fasting state in between 8 a.m. to 9 a.m. Plasma was separated by centrifugation and stored at $-20{ }^{\circ} \mathrm{C}$ until assay. Serum LH, FSH and total testosterone (TT) were measured by chemiluminescent microparticle immunoassay with Seimens ADVIA Centaur XP (USA). LH-FSH ratio > 1.0 was considered as altered and serum TT $>46 \mathrm{ng} / \mathrm{dL}$ was considered as hyperandrogenemia $[9,10]$.

\section{Statistical analysis}

Data were analyzed by SPSS version 22.0. Quantitative data were expressed as mean \pm standard deviation (SD) or median (interquartile range (IQR)) depending on their distribution and qualitative data were expressed with frequency (\%). In this study, all the data were normally distributed except LH-FSH ratio which was positively skewed. To see the association between groups of LH-FSH ratio status and androgen status, independent samples $t$-test or Mann-Whitney $\mathrm{U}$ test and one-way analysis of variance (ANOVA) or Kruskal-Wallis test were done for quantitative values as appropriate and Chi-square test was done for qualitative variables. Spearman's correlation test was done to see the correlation between LH-FSH ratio with se- rum TT. Binary logistic regression analysis was done to see the association of altered LH-FSH ratio and hyperandrogenemia with clinical and biochemical variables. Receiver-operating characteristic (ROC) curve was analyzed to see whether serum LH-FSH ratio could discriminate between the two androgen statuses.

\section{Results}

This cross-sectional study included 550 reproductive aged females (age range: 13 - 39 years, mean $\pm \mathrm{SD}, 23.14 \pm 4.80$ years; BMI: mean $\pm \mathrm{SD}, 27.64 \pm 5.34 \mathrm{~kg} / \mathrm{m}^{2}$; significant hirsutism: $82.36 \%$ ) of PCOS. More than two-thirds (70.73\%) of them had altered LH-FSH ratio with a cut-off $>1.0$. However, there were no significant differences of the frequencies of any clinical or laboratory variables between the two groups of LH-FSH ratio (NS for all). In contrast, several personal (menstrual abnormality: $88.5 \%$ vs. $78.2 \%, \mathrm{P}=0.002$ ) and family histories (PCOS: $24.4 \%$ vs. $17.4 \%, \mathrm{P}=0.045$; diabetes mellitus $(\mathrm{DM}): 67.5 \%$ vs. $57.3 \%, \mathrm{P}=0.017$; obesity: $62.0 \%$ vs. $50.6 \%, \mathrm{P}=0.009)$ and significant hirsutism $(91.5 \%$ vs. $75.6 \%, \mathrm{P}<0.001)$ were present with significantly higher frequency in women with hyperandrogenemia defined as serum TT $>46 \mathrm{ng} / \mathrm{dL}$ (Table 1).

Patients with altered LH-FSH were younger $(22.77 \pm 4.77$ vs. $24.01 \pm 4.79$ years; $\mathrm{P}=0.006)$ and had significantly higher serum TT (51.47 \pm 29.92 vs. $44.56 \pm 29.07 \mathrm{ng} / \mathrm{dL}, \mathrm{P}=0.013)$ than those with normal LH-FSH. None of the other variables (age, BMI, WC, systolic blood pressure (SBP) and diastolic blood pressure (DBP)) showed any statistically significant difference between the two groups of LH-FSH ratio (NS for all). On the other hand, the mean value of SBP (112.72 \pm 13.16 vs. 110.37 $\pm 13.21 \mathrm{~mm} \mathrm{Hg}, \mathrm{P}=0.039)$ and $\mathrm{DBP}(76.50 \pm 8.90$ vs. 74.23 $\pm 9.68 \mathrm{~mm} \mathrm{Hg}, \mathrm{P}=0.005$ ) were significantly higher in PCOS women with hyperandrogenemia. But other variables (age, BMI and WC) including LH-FSH ratio were not statistically different between the two androgen statuses (NS for all) (Table 2).

In Figure 1, serum TT (mean $\pm \mathrm{SD}, \mathrm{ng} / \mathrm{dL}$ ) of patients with PCOS is shown among different altered LH-FSH ratios and compared with normal LH-FSH ratio defined by $\leq 1.0$. The mean values of serum TT (ng/dL) were significantly higher in all altered LH-FSH ratio groups $(\leq 1.0(44.56 \pm 29.07)$ vs. $>1.0$ $(51.47 \pm 29.92, \mathrm{P}=0.013)$ vs. $>1.5(53.02 \pm 29.91, \mathrm{P}=0.004)$ vs. $>2.0(55.89 \pm 30.21, \mathrm{P}=0.010)$ vs. $>2.5(53.03 \pm 30.12, \mathrm{P}$ $=0.020)$ vs. $>3.0(54.92 \pm 30.41, \mathrm{P}=0.013)$ vs. $>3.5(57.69$ $\pm 31.12, \mathrm{P}=0.005)$ ). An increasing trend of serum testosterone value with higher cut-offs of LH-FSH status was also observed.

The mean value of serum TT became higher progressively from first to fourth quartile. In post hoc analysis, the serum TTs $(\mathrm{ng} / \mathrm{dL})$ of third (third vs. first: $52.45 \pm 29.87$ vs. $44.18 \pm 28.76$; $\mathrm{P}=0.022$ ) and fourth (fourth vs. first: $53.0 \pm 30.24$ vs. 44.18 $\pm 28.76 ; \mathrm{P}=0.015)$ quartiles were significantly higher than the first quartile (Fig. 2). Similarly, the median value of LHFSH ratio was higher in the second to fourth quartiles (median (IQR): $\mathrm{Q}_{1}(1.25(0.72-1.99))$ vs. $\mathrm{Q}_{2}(1.50(1.04-2.40), \mathrm{P}=$ $0.033)$ vs. $Q_{3}(1.80(0.88-2.48), P=0.009)$ vs. $Q_{4}(1.66(1.00$ - 2.36), $\mathrm{P}=0.031)$ ) than the first quartile of serum TT (Fig. 3).

As shown in Figure 4, the correlation between LH-FSH 


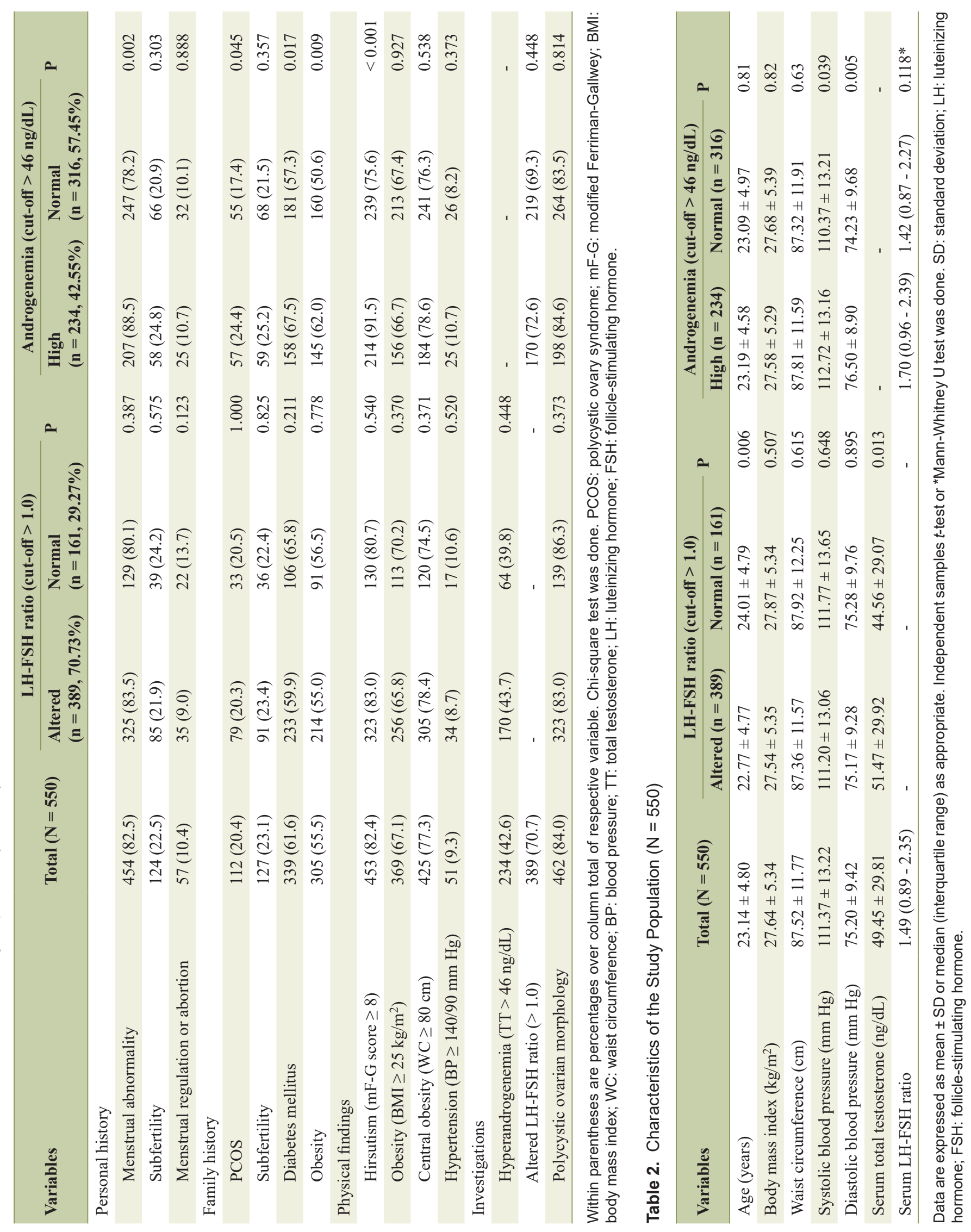




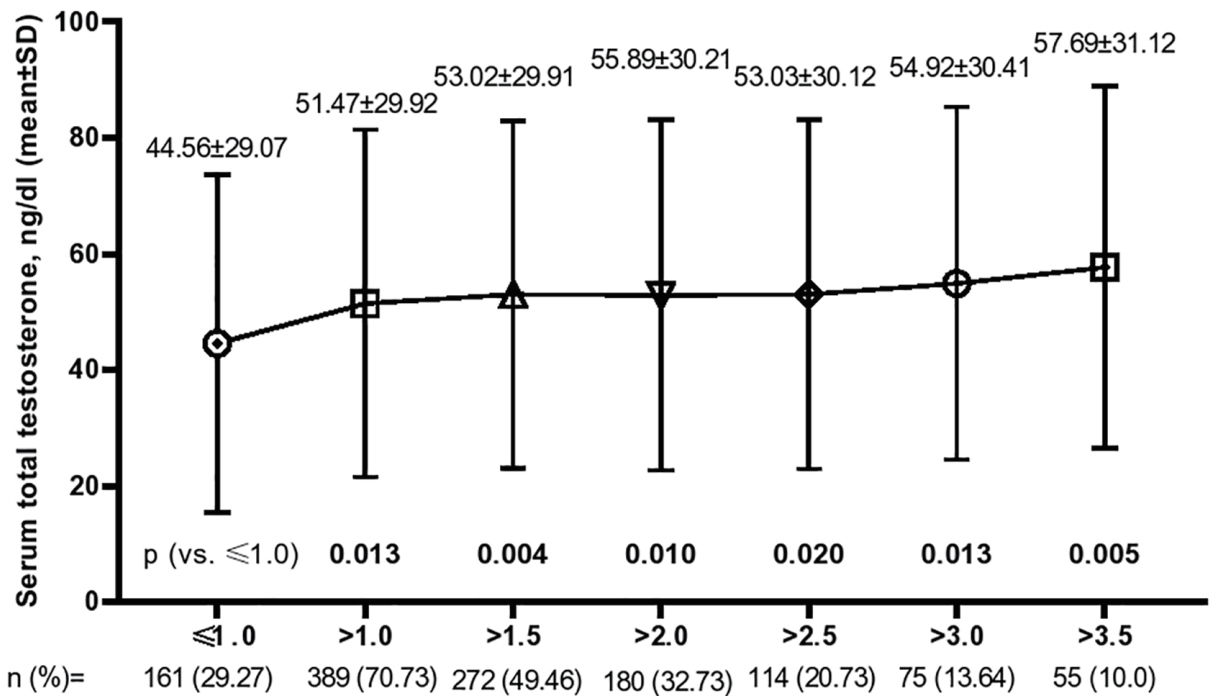

Various cutoffs of LH-FSH ratio

Independent samples- $\mathrm{T}$ test was done

Figure 1. Serum total testosterone (mean $\pm \mathrm{SD}$ ) in altered LH-FSH ratio with different cut-offs. SD: standard deviation; LH: luteinizing hormone; FSH: follicle-stimulating hormone.

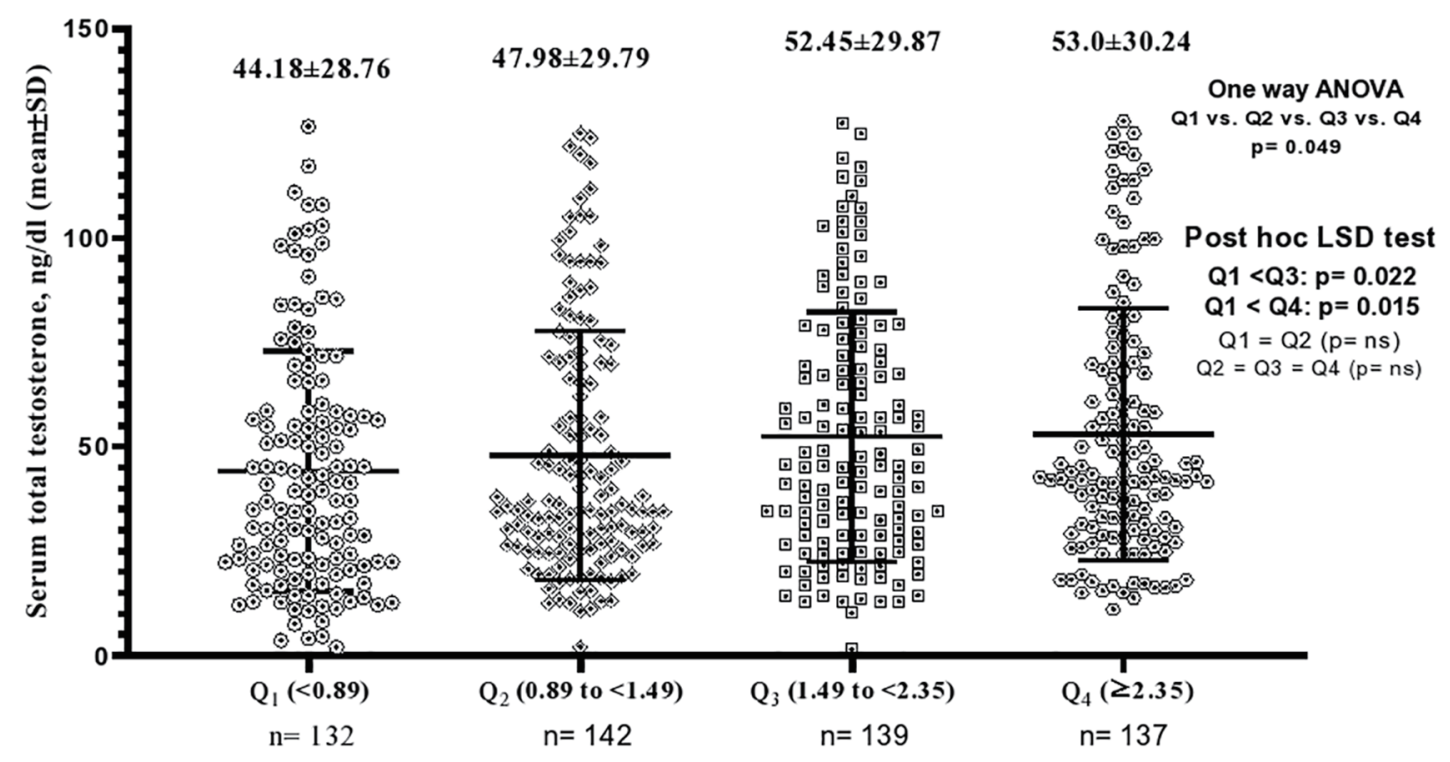

\section{Quartiles of LH-FSH ratio}

One way ANOVA with post hoc LSD test was done

Figure 2. Serum total testosterone level (mean $\pm \mathrm{SD}$ ) according to the quartiles of LH-FSH ratio. SD: standard deviation; LH: luteinizing hormone; FSH: follicle-stimulating hormone. 


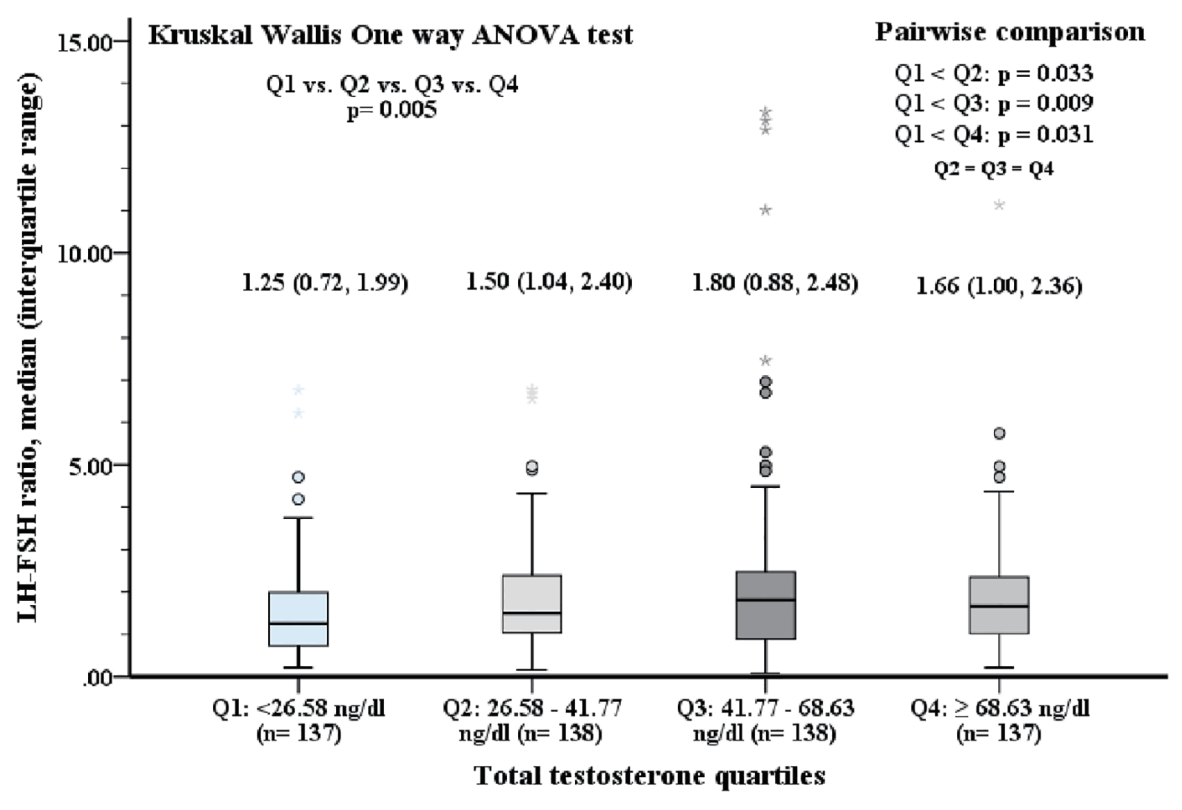

Kruskal Wallis one way ANOVA test with pair wise comparison was done

Figure 3. Serum LH-FSH ratio according to quartiles of serum total testosterone. LH: luteinizing hormone; FSH: follicle-stimulating hormone.

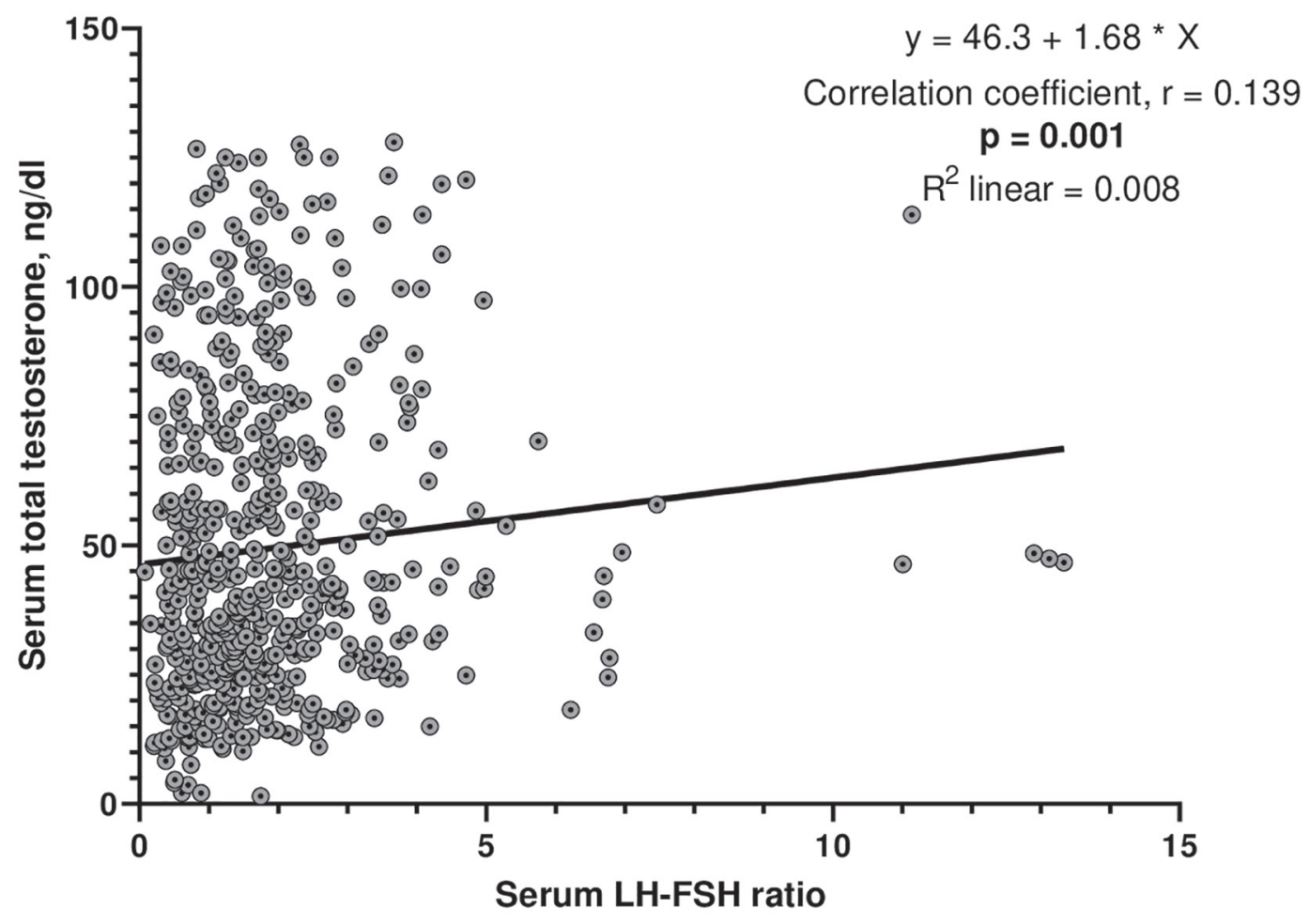

Spearman's correlation test was done

Figure 4. Correlation of serum total testosterone with LH-FSH ratio. LH: luteinizing hormone; FSH: follicle-stimulating hormone. 
Table 3. Multivariable Logistic Regression Analysis for Altered LH-FSH Ratio (Cut-Off $>1.0)$ and Hyperandrogenemia (Cut-Off $>46$ $\mathrm{ng} / \mathrm{dL}$ ) in the Study Population

\begin{tabular}{|c|c|c|c|c|c|c|}
\hline \multirow{2}{*}{ Covariates } & \multicolumn{2}{|c|}{ Altered LH-FSH ratio } & \multirow{2}{*}{$\mathbf{P}$} & \multicolumn{2}{|c|}{ Hyperandrogenemia } & \multirow{2}{*}{$\mathbf{P}$} \\
\hline & B & OR $(95 \%$ CI $)$ & & B & OR $(95 \%$ CI $)$ & \\
\hline Age, years & -0.053 & $0.948(0.911-0.986)$ & 0.008 & 0.002 & $1.002(0.965-1.040)$ & 0.921 \\
\hline Waist circumference, $\mathrm{cm}$ & 0.007 & $1.007(0.976-1.038)$ & 0.671 & 0.011 & $1.011(0.983-1.041)$ & 0.434 \\
\hline Systolic blood pressure, $\mathrm{mm} \mathrm{Hg}$ & -0.004 & $0.996(0.977-1.015)$ & 0.682 & 0.003 & $1.003(0.985-1.021)$ & 0.774 \\
\hline Serum total testosterone, $\mathrm{ng} / \mathrm{dL}$ & 0.082 & $1.086(1.016-1.160)$ & 0.016 & - & - & - \\
\hline Serum LH-FSH ratio & - & - & - & 0.137 & $1.147(1.025-1.284)$ & 0.017 \\
\hline
\end{tabular}

Binary logistic regression analysis was done. OR: odds ratio; Cl: confidence interval; LH: luteinizing hormone; FSH: follicle-stimulating hormone.

ratio and serum TT was positive and statistically significant $(\mathrm{r}=0.139, \mathrm{P}=0.001)$. Logistic regression revealed that age (odds ratio (OR) $(95 \%$ confidence interval $(\mathrm{CI}))$ : $0.948(0.911$ - 0.986), $\mathrm{P}=0.008)$ and serum TT (OR (95\% CI): 1.086 (1.016 - 1.160), $\mathrm{P}=0.016)$ had significant predictive associations with altered LH-FSH ratio in PCOS. On the other hand, DBP (OR $(95 \% \mathrm{CI}): 1.028(1.002-1.054), \mathrm{P}=0.036)$ and $\mathrm{LH}-\mathrm{FSH}$ ratio (OR (95\% CI): 1.147 (1.025 - 1.284), P =0.017) were associated predictors for high androgen status in women with PCOS (Table 3). ROC curve analysis showed that LH-FSH ratio could not discriminate hyperandrogenemia from normoandrogenemia (area under the curve $=0.54, \mathrm{P}=0.118$ ) in women with PCOS (not shown).

\section{Discussion}

This study was done encompassing 550 women with PCOS and showed that about $71 \%$ had an altered LH-FSH ratio with the cut-off $>1.0$ and $42.55 \%$ had hyperandrogenemia with a cut-off TT $>46 \mathrm{ng} / \mathrm{dL}$. Altered LH-FSH was negatively associated with the age of the PCOS patients, and was positively associated with serum TT level. Hyperandrogenemia was positively associated with both DBP and serum LH-FSH ratio. A significant correlation between LH-FSH ratio and serum TT level was observed. However, it was not possible to distinguish between hyperandrogenemia and normoandrogenemia by using the LH-FSH ratio.

Altered LH-FSH ratio due to increased gonadotrophinreleasing hormone $(\mathrm{GnRH})$ pulse frequency is a characteristic feature of PCOS. Normally the ratio is $1: 1$. In PCOS, the LH value exceeds FSH causing excess ovarian androgen production and ovulatory dysfunction. Various cut-offs of LH-FSH ratio were proposed. However the cut-off $>1.0$ was found to be the best in diagnosing PCOS considering both sensitivity and specificity $[8,9]$. However, its utility was not found by all and also was not included in the diagnostic criteria of PCOS [11].

We found altered LH-FSH ratio in around $71 \%$ of our study participants which was similar to a study finding [8]. The frequency actually depends on the cut-off used to define the altered status. A previous study from Bangladesh found 58.2\% of 55 PCOS patients with altered LH-FSH ratio (cut-off $>1.0$ )
[12]. The mean age of PCOS patients of our study was lower than that previous study. As serum FSH increases with age and LH remains stable, so the ratio may decrease with age $[13,14]$. We also found similar association with age. The frequency of altered LH-FSH ratio reduced to $32.73 \%$ with the cutoff of $>$ 2.0. This frequency was lower than other studies $[15,16]$. As PCOS patients from south Asian background have higher frequency of obesity, they have lower LH-FSH ratio [17]. However, we did not find any association of LH-FSH ratio with BMI which was similar to a study finding [18]. A meta-analysis found significant association of metabolic features with hyperandrogenism in PCOS [19]. In our study, the metabolic manifestations were more frequent with hyperandrogenemia rather than altered LH-FSH ratio. Similarly, another study did not find any correlation between different biochemical parameters related to insulin resistance with altered LH-FSH ratio $[16,20]$.

Around $82.36 \%$ of women with PCOS had significant manifestation of hirsutism, but $43 \%$ had hyperandrogenemia and significantly higher frequency of hirsutism was observed with hyperandrogenemia group. The discrepancy between the clinical and biochemical frequency of androgen status may be due to the local conversion of testosterone to dihydroxytestosterone by $5 \alpha$-reductase. South Asian women with PCOS have more hirsutism despite a similar serum testosterone level than other races [21]. A study conducted in an Endocrine clinic among Sri Lankan women with PCOS found around 75\% patients with hirsutism and $34 \%$ with hyperandrogenemia with a cut-off of TT of $3 \mathrm{nmol} / \mathrm{L}$ (about $86.5 \mathrm{ng} / \mathrm{dL}$ ) [22]. We found an association between DBP with hyperandrogenemia even after adjustment of age and BMI. A study conducted on Taiwanese women with PCOS also found an association of hyperandrogenemia with hypertension [23]. Higher blood pressure as a component of metabolic syndrome is common in PCOS and is associated with increased risk of atherosclerotic cardiovascular disease [24]. Increased vascular stiffness and endothelial dysfunction are responsible for elevated blood pressure which might be associated with hyperandrogenemia irrespective of insulin resistance $[25,26]$. We did not study the insulin resistance here.

We found that serum TT was associated with altered LHFSH ratio in PCOS. Compared with normal LH-FSH, serum 
TT progressively increased in altered LH-FSH ratio with higher cut-offs. Two studies from India also found similar findings $[27,28]$. These findings also indicate that $\mathrm{LH}-\mathrm{FSH}$ ratio just $>1.0$ is enough to produce significant testosterone from the ovaries causing hyperandrogenemia. However, altered LHFSH ratio holding cut-off at 1.0 was not associated with androgen status in women with PCOS as revealed by the findings of statistically insignificant difference of frequency of hyperandrogenism between altered and normal LH-FSH ratio groups. Therefore, the cut-off used for LH-FSH ratio that would better discriminate hyperandrogenic status may need to be reconsidered in future.

This study included a relatively large sample size. It demonstrated a significant correlation of LH-FSH ratio with TT of PCOS patients from South Asian background where metabolic features are predominant. However, we have no populationspecific data of reference range of serum TT. Besides, we did not measure serum sex hormone binding globulin to calculate free testosterone index or free testosterone which might have correlated better with androgen status that could not be assessed in this study [1].

\section{Conclusions}

LH-FSH ratio was significantly correlated with biochemical androgen status in women with PCOS from Bangladesh. This study finding will hopefully help to understand the pathogenesis and management of hyperandrogenism in PCOS from South Asian background more efficiently.

\section{Acknowledgments}

We appreciate Department of Biochemistry and Microbiology, BSMMU for biochemical and hormonal assay.

\section{Financial Disclosure}

Partial funding support by the Research and Development of BSMMU.

\section{Conflict of Interest}

None of the authors have any conflict of interest to declare.

\section{Informed Consent}

Written informed consent was taken from each participant before enrollment.

\section{Author Contributions}

MSM, HB and MAH planned the study protocol, analyzed data and wrote the manuscript. All other authors collected data and involved directly in patient management. All the authors read the manuscript and approved for publication.

\section{Data Availability}

The data that supported the study findings can be shared on reasonable ground from the corresponding author.

\section{References}

1. Teede H, Misso M, Costello M, Dokras A, Laven J, Moran $\mathrm{L}$, et al. International evidence-based guideline for the assessment and management of polycystic ovary syndrome 2018. National Health and Medical Research Council (NHMRC). 2018:1-198. Available at: https://www. monash.edu/_data/assets/pdf_file/0004/1412644/PCOS Evidence-Bāsed-Guidelines_20181009.pdf.

2. $\bar{R}$ Rodin DA, Bano G, Bland JM, Taylor K, Nussey SS. Polycystic ovaries and associated metabolic abnormalities in Indian subcontinent Asian women. Clin Endocrinol (Oxf). 1998;49(1):91-99.

3. Rotterdam Eshre Asrm-Sponsored Pcos Consensus Workshop Group. Revised 2003 consensus on diagnostic criteria and long-term health risks related to polycystic ovary syndrome. Fertil Steril. 2004;81(1):19-25.

4. Coutinho EA, Kauffman AS. The role of the brain in the pathogenesis and physiology of polycystic ovary syndrome (PCOS). Med Sci (Basel). 2019;7(8):84.

5. Ashraf S, Nabi M, Rasool S ul A, Rashid F, Amin S. Hyperandrogenism in polycystic ovarian syndrome and role of CYP gene variants: a review. Egypt J Med Hum Genet. 2019;20(1):25.

6. World Health Organization. The Asia-Pacific perspective: Redefining obesity and its treatment. Regional Office for the Western Pacific. 2000. https://apps.who.int/iris/handle/10665/206936.

7. Williams B, Mancia G, Spiering W, Rosei EA, Azizi M, Burnier M, Clement DL, et al. [2018 ESC/ESH Guidelines for the management of arterial hypertension]. Kardiol Pol. 2019;77(2):71-159.

8. Escobar-Morreale HF, Carmina E, Dewailly D, Gambineri A, Kelestimur F, Moghetti P, Pugeat M, et al. Epidemiology, diagnosis and management of hirsutism: a consensus statement by the Androgen Excess and Polycystic Ovary Syndrome Society. Hum Reprod Update. 2012;18(2):146-170.

9. Hsu MI, Liou TH, Liang SJ, Su HW, Wu CH, Hsu CS. Inappropriate gonadotropin secretion in polycystic ovary syndrome. Fertil Steril. 2009;91(4):1168-1174.

10. Tola H, Abbas M, Alhassan EA, Shrif NE, Rida M. Assessment of the role of the anti-Mullerian hormone, luteinizing hormone/follicle stimulating hormone ratio in the diagnosis of polycystic ovary syndrome in Sudanese women. Open Access Maced J Med Sci. 2018;6(7):12441247.

11. Braunstein GD, Reitz RE, Buch A, Schnell D, Caul- 
field MP. Testosterone reference ranges in normally cycling healthy premenopausal women. J Sex Med. 2011;8(10):2924-2934.

12. Cho LW, Jayagopal V, Kilpatrick ES, Holding S, Atkin $\mathrm{SL}$. The LH/FSH ratio has little use in diagnosing polycystic ovarian syndrome. Ann Clin Biochem. 2006;43(Pt 3):217-219.

13. Nahar K, Yasmin H, Pramanik L. Study of Polycystic Ovaries (PCO) in Mymensingh Medical College Hospital, Bangladesh. J Bangladesh Coll Physicians Surg. 2015;32(3):142-148.

14. Racoubian E, Aimagambetova G, Finan RR, Almawi WY. Age-dependent changes in anti-Mullerian hormone levels in Lebanese females: correlation with basal FSH and LH levels and LH/FSH ratio: a cross-sectional study. BMC Womens Health. 2020;20(1):134.

15. Bohlke K, Cramer DW, Barbieri RL. Relation of luteinizing hormone levels to body mass index in premenopausal women. Fertil Steril. 1998;69(3):500-504.

16. Alnakash AH, Al-Tae'e NK. Polycystic ovarian syndrome: The correlation between the LH/FSH ratio and disease manifestations. Middle East Fertil Soc J. 2007;12(1):3540. Available at: http://www.bioline.org.br/pdf?mf07006.

17. Banaszewska B, Spaczynski RZ, Pelesz M, Pawelczyk L. Incidence of elevated LH/FSH ratio in polycystic ovary syndrome women with normo- and hyperinsulinemia. Rocz Akad Med Bialymst. 2003;48:131-134.

18. Saadia Z. Follicle Stimulating Hormone (LH: FSH) Ratio in Polycystic Ovary Syndrome (PCOS) - Obese vs. NonObese Women. Med Arch. 2020;74(4):289-293.

19. Yang R, Yang S, Li R, Liu P, Qiao J, Zhang Y. Effects of hyperandrogenism on metabolic abnormalities in patients with polycystic ovary syndrome: a meta-analysis. Reprod Biol Endocrinol. 2016;14(1):67.

20. Park $\mathrm{CH}$, Chun S. Association between serum gonadotropin level and insulin resistance-related parameters in Korean women with polycystic ovary syndrome. Obstet
Gynecol Sci. 2016;59(6):498-505.

21. Wijeyaratne CN, Balen AH, Barth JH, Belchetz PE. Clinical manifestations and insulin resistance (IR) in polycystic ovary syndrome (PCOS) among South Asians and Caucasians: is there a difference? Clin Endocrinol (Oxf). 2002;57(3):343-350.

22. Wijeyaratne CN, Seneviratne Rde A, Dahanayake S, Kumarapeli V, Palipane E, Kuruppu N, Yapa C, et al. Phenotype and metabolic profile of South Asian women with polycystic ovary syndrome (PCOS): results of a large database from a specialist Endocrine Clinic. Hum Reprod. 2011;26(1):202-213.

23. Chen MJ, Yang WS, Yang JH, Chen CL, Ho HN, Yang YS. Relationship between androgen levels and blood pressure in young women with polycystic ovary syndrome. Hypertension. 2007;49(6):1442-1447.

24. Holte J, Gennarelli G, Berne C, Bergh T, Lithell H. Elevated ambulatory day-time blood pressure in women with polycystic ovary syndrome: a sign of a pre-hypertensive state? Hum Reprod. 1996;11(1):23-28.

25. Kelly CJ, Speirs A, Gould GW, Petrie JR, Lyall H, Connell JM. Altered vascular function in young women with polycystic ovary syndrome. J Clin Endocrinol Metab. 2002;87(2):742-746.

26. Paradisi G, Steinberg HO, Hempfling A, Cronin J, Hook G, Shepard MK, Baron AD. Polycystic ovary syndrome is associated with endothelial dysfunction. Circulation. 2001;103(10):1410-1415.

27. Malini NA, Roy George K. Evaluation of different ranges of LH:FSH ratios in polycystic ovarian syndrome (PCOS) - Clinical based case control study. Gen Comp Endocrinol. 2018;260:51-57.

28. Suresh S, Vijayakumar T. Correlations of insulin resistance and serum testosterone levels with LH:FSH ratio and oxidative stress in women with functional ovarian hyperandrogenism. Indian J Clin Biochem. 2015;30(3):345350 . 